\title{
Qualidade de vida pós-transplante renal: revisão integrativa
}

\author{
Reginaldo Passoni dos Santos, Daniele Lais Brandalize Rocha \\ Pontificia Universidad Católica Del Paraná. Brasil
}

\section{Resumo}

Buscaran-se evidências disponíveis na literatura sobre a melhoria na qualidade de vida de pacientes após o transplante renal. Trata-se de uma revisão integrativa, realizada por meio de busca de artigos indexados nas bases de dados LILACS (Literatura Latino-americana e do Caribe em Ciências da Saúde), BDENF (Base de Dados da Enfermagem), SciELO (Scientific Eletronic Library Online) e PubMed. Os critérios de inclusão foram: pesquisa primária; texto em português, inglês ou espanhol; publicação entre o período de janeiro de 2002 a dezembro de 2012. De modo geral, o transplante melhora expressivamente a qualidade de vida do paciente com renal crônico, sendo prevalente a melhora relacionada ao domínio físico. Contudo, identificaram baixos escores referente às de dimensões/domínios "relação social", "meio ambiente", "nível de dependência" e "aspectos psicológicos". Confirmou-se que o transplante é responsável pela melhoria da qualidade de vida do paciente renal crônico pós procedimento, entretanto, sugere-se que mais pesquisas sejam realizadas.

\section{DESCRITORES:}

- QUALIDADE DE VIDA

- TRANSPLANTE DE RIM

- QUESTIONÁRIOS

- ENFERMAGEM
Correspondencia:

Reginaldo Passoni dos Santos

Union Avenue, 500

Becker Village

CP- 85902-532. Brazil

E-mail: regi-pas@hotmail.com
Quality of life after kidney transplantation: review integrative

\begin{abstract}
We sought evidence available in the literature on improving the quality of life of patients after renal transplantation. It is an integrative review, conducted through search of articles indexed in the databases LILACS (Latin American and Caribbean Health Sciences), BDENF (Database of Nursing), SciELO (Scientific Electronic Library Online ) and PubMed. Inclusion criteria were: primary research, text in Portuguese, English or Spanish; published between the period January 2002 to December 2012. In general, transplantation significantly improves the quality of life of patients with CKD is prevalent improvement related to the physical domain. However, we found low scores regarding dimensions / domains on "social relationship", "environment", "degree of dependence" and "psychological aspects". It was confirmed that the transplant is responsible for the improvement in the quality of life of chronic renal patient post procedure, however, it is $s$ more research needs to be done.
\end{abstract}

\section{DESCRIPTORS:}

- QUALITY OF LIFE

- KIDNEYTRANSPLANTATION

- QUESTIONNAIRES

- NURSING

\section{$\bullet \bullet \bullet \bullet \bullet \bullet \bullet \bullet \bullet \bullet \bullet \bullet \bullet \bullet \bullet \bullet \bullet \bullet \bullet \bullet$ Calidad de vida tras el trasplante de riñón: revisión integral}

\section{Resumen}

Se buscaron pruebas disponibles en la literatura sobre la mejora de la calidad de vida de los después del trasplante renal. Se trata de una revisión integradora, levando a cabo a través de la búsqueda de artículos indexados en las bases de datos LILACS (Literatura La- 
tinoamericada y del Caribe en Ciencias), BDENF (Base de Datos de Enfermería), SciELO (Scientific ELetronic Library Online) y PubMed. Los criterios de inclusión fueron: investivatión primaria, el texto en portugués, inglés o español, publicados entre el período de enero 2002 a diciembre 2012. En gerenal el trasplante mejora significativamente la calidad de vida de los pacientes con enfermedad renal crónica es prevalente mejora relacionada con el dominio físico. Sin embargo, encontramos bajas puntuaciones en las dimensiones "relación social", "medio ambiente", "grado de dependencia" y "psicológica". Se confirmó que el trasplante es responsable de la adición de la calidad de vida de la pacientes después del procedimiento renal crónica, sin embargo, sugiere que haga más investigación.

\section{PALABRAS CLAVE:}

- CALIDAD DE VIDA

- TRASPLANTE DE RIÑÓN

- CUESTIONARIOS

- ENFERMERÍA

\section{Introdução}

Em saúde e, não obstante, em Enfermagem, a Prática Baseada em Evidências (PBE) é fortemente encorajada, isso, porque se acredita que a aplicação das melhores evidências científicas na prática profissional permite mais rigor e agilidade no diagnóstico, escolha da melhor abordagem terapêutica a cada indivíduo fomentada por resultados de pesquisas clínicas e adoção de medidas preventivas mais eficazes ${ }^{(1)}$. Tais contribuições garantem a minimização dos gastos efetivos, o aprimoramento de técnicas com possibilidade de recuperação precoce do cliente/paciente e suporte científico às decisões clínicas para intervenção antecipada relacionada com possíveis desfechos negativos ${ }^{(1-2)}$. Ressalta-se que, tal como todos os outros, este modelo de assistência deve ser utilizado respeitando-se os princípios dispostos no Código de Ética Profissional (CEP), instituído pelo Conselho Internacional de Enfermeiras (os) (CIE) ${ }^{(3)}$. Assim, enfatiza-se que, a partir das contribuições apontadas, os cuidados estabelecidos por meio da PBE aspiram à melhoria da condição de saúde do ser doente, esperandose que, por conseguinte, haja melhoria da qualidade de vida (QV) deste ${ }^{(1)}$.

Seguindo o disposto, cabe lembrar que de entre os fatores determinantes para o aumento da longevidade da população humana, citam-se a adoção de hábitos de vida saudáveis, melhoria nas políticas de previdência e o avanço da tecnologia em saúde. Contudo, um desa- fio emergido da expansão na expectativa de vida é a promoção e manutenção da vitalidade do ser humano. Esse desafio apresenta-se ainda maior, quando se trata da promoção da QV do indivíduo portador de Doença Crônica Não Transmissível (DCNT) ${ }^{(4)}$.

A complexidade, bem como o dimensionamento da inferência da condição clínico-patológica no cotidiano da pessoa com doença crônica, impulsiona um número cada vez maior de profissionais da Enfermagem que efetivam seu exercício baseando-se em evidências provenientes de resultados de estudos científicos, sendo estes constituídos como sustentáculos das condutas de promoção da QV do ser enfermo ${ }^{(4)}$. Porém, para que haja a perspectiva do planejamento estratégico de cuidados que abarque ações de promoção da QV é preciso, inicialmente, buscar conhecimento sobre o estado da arte.

No universo das pesquisas sobre este tema, conduzidas com grupos específicos, a divulgação de investigações realizadas com portadores de doença renal crônica (DRC) em estágio terminal mostra-se, consideravelmente, ampla na literatura cientifica brasileira, com especial atenção aos portadores de DRC em hemodiálise $(H D)^{(5-7)}$. Entre os motivos apontados para a prevalência de inquéritos com este grupo de nefropatas, está o fato de que a QV da pessoa com DRC em HD mostra-se evidentemente comprometida, sendo necessário conhecer a amplitude desse comprometimento e o nível de interferência no tratamento, possibilitando, a partir disso, a incorporação prática de medidas que propiciem a minimização da problemática ${ }^{(7-8)}$.

Com anseio de melhorar sua $\mathrm{QV}$, a maioria dos clientes em tratamento hemodialítico, se não todos, carrega consigo o desejo de um dia se submeterem ao procedimento cirúrgico mais esperado de suas vidas, o transplante (Tx) renal. Dessa maneira, infere-se que o Tx seja a carta de alforria de todos os que realizam terapia renal substitutiva (TRS) por meio da HD. Esse intenso sentimento de fé e esperança pela realização de um Tx renal, desvelado na narrativa de clientes em HD e, apresentado por diversos autores, é naturalmente compreendido, pois, o doente renal crônico transplantado é visto como aquele que vivenciou todas as atribulações, sofrimentos e desafios impostos pela patologia e ainda assim, simbolicamente, venceu até mesmo a própria doença ${ }^{(6-8)}$.

Frente à compreensão natural dos sentimentos aflorados pelo doente renal crônico em hemodiálise e às histórias de superação do transplantado renal, apresenta-se como objetivo do estudo agora desenvolvido identificar 
quais as evidências disponíveis na literatura sobre a melhoria na qualidade de vida de paciente com doença renal crônica após o transplante renal.

A escolha do tema justifica-se no anseio dos pesquisadores em trazer à luz do conhecimento as evidências na melhoria da QV que o paciente com DRC apresenta após o Tx renal. Na mesma direção, salienta-se ainda a pretensão de identificar na literatura se há possíveis dimensões relacionadas à $Q V$ no pós $T x$ renal com baixos escores de avaliação, uma vez que a realização do transplante pauta-se, não apenas no restabelecimento de funções orgânicas vitais, mas, principalmente, na atribuição de melhoria à QV do paciente com DRC terminal. Por conta disso, estabeleceu-se a seguinte questão norteadora: Quais são as evidências disponíveis na literatura sobre a melhoria na qualidade de vida de paciente com doença renal crônica após o transplante renal?

\section{Metodología}

A PBE pauta-se no conceito de "uso consciencioso, explícito e criterioso de teorias [...]", aplicando-se na prática as evidências que melhor atendem às necessidades de cuidado individual ou coletivo ${ }^{(9: 550)}$. Assim, este trabalho integra uma revisão integrativa a qual fornece subsídios valiosos para a PBE em Enfermagem e saúde, pois, permite sintetizar e reunir a literatura sobre a melhoria na qualidade de vida de paciente com DRC após o Tx renal em um único estudo, tornando-se instrumento de validação para iniciativas de práticas profissionais baseadas em evidências científicas. Cabe ressaltar, que a elaboração da revisão integrativa deve seguir determinadas recomendações práticas, com o intuito de alcançar a mesma qualidade técnicocientífica exigida em pesquisas de campo provenientes do rigor metodológico estabelecido(10).

Seguindo o disposto, este estudo contemplou as seguintes fases: escolha do tema e elaboração da questão norteadora; busca dos estudos nas bases de dados com aplicação de critérios de inclusão e exclusão; extração, organização, sumarização e análise das informações de acordo com o nível de evidência; discussão dos resultados e apresentação da revisão propriamente dita(11).

Foram realizadas buscas de artigos indexados nas bases de dados LILACS (Literatura Latino-americana e do Caribe em Ciências da Saúde), BDENF (Base de Dados da Enfermagem), SciELO (Scientific Eletronic Library Online) e PubMed, sendo esta por meio de acesso à Biblioteca Nacional de Medicina dos Estados Unidos.
Utilizaram-se os seguintes critérios de inclusão: pesquisa primária; texto em português, inglês ou espanhol; publicação entre o período de janeiro de 2002 a dezembro de 2012. A busca ocorreu durante o mês de fevereiro de 2013, por meio da utilização conjunta dos Descritores em Ciências da Saúde-DeCS ("qualidade de vida"; "transplante de rim"; "questionários") e Medical Subject Headings-MeSH ("quality of life"; "kidney transplantation"; "questionnaries"), através da pesquisa booleana (AND/ OR). A utilização dos DeCS e MeSH nas bases de dados conforme descrito, gerou a localização de 356 estudos. Deste total, foram excluídos por meio da leitura do título $(n=326)$, do resumo $(n=12)$ e do texto na íntegra $(n=7)$ todos os artigos que não atenderam aos critérios de inclusão e, principalmente, aqueles que não contemplavam uma resposta clara e objetiva à questão norteadora.

Ressalta-se que, mesmo atendendo aos critérios de inclusão, bem como à questão norteadora, alguns artigos foram excluídos desta revisão por impossibilidade de acesso ao texto na íntegra.

A identificação dos níveis de evidência (NE), baseou-se na categorização dos estudos de acordo com a classificação apresentada no quadro 1.

Quadro 1. Classificação dos níveis de evidência ${ }^{12}$

\begin{tabular}{|c|l|}
\hline Nível & Descrição da evidência \\
\hline I & $\begin{array}{l}\text { Evidência oriunda de revisão sistemática e/ou metanálise } \\
\text { na qual há inclusão somente de estudos clínicos controlados } \\
\text { e randomizados com delineamento adequado; }\end{array}$ \\
\hline II & $\begin{array}{l}\text { Evidência oriunda de, no mínimo, um estudo clínico } \\
\text { controlado e randomizado com delineamento adequado; }\end{array}$ \\
\hline III & $\begin{array}{l}\text { Evidência oriunda de um único estudo controlado e } \\
\text { randomizado com delineamento adequado; }\end{array}$ \\
\hline IV & $\begin{array}{l}\text { Evidência oriunda de um estudo de caso-controle ou coorte } \\
\text { com delineamento adequado; }\end{array}$ \\
\hline V & $\begin{array}{l}\text { Evidência oriunda de revisão sistemática de estudos } \\
\text { descritivos e qualitativos com delineamento adequado; }\end{array}$ \\
\hline VI & $\begin{array}{l}\text { Evidência oriunda de apenas um estudo descritivo e } \\
\text { qualitativo com delineamento adequado; }\end{array}$ \\
\hline VII & $\begin{array}{l}\text { Evidência oriunda de reflexões de autoridades e/ou } \\
\text { relatórios elaborados por grupos de especialistas. }\end{array}$
\end{tabular}

\section{Resultados}

Foram identificados 11 artigos que atenderam tanto aos critérios de inclusão quanto ao objetivo deste estudo. Destes, cinco ${ }^{(13-17)}$ apresentavam investigações científicas desenvolvidas na Europa, quatro ${ }^{18-21}$ na América do Sul, um ${ }^{(22)}$ nos Estados Unidos da América (EUA) e um ${ }^{(23)}$ em África. Com relação ao questionário aplicado para a 
coleta dos dados, observou-se que cinco ${ }^{(16-17,19-20,22)}$ estudos aplicaram o instrumento genérico Short Form-36 (SF-36), dois ${ }^{(18,21)}$ o World Health Organization Quality of Life (WHOQOL), três ${ }^{(13-15)}$ fizeram uso associado do SF-36 com outro (s) questionário (s) e um ${ }^{(23)}$ estudo utilizou a Escala de Status de desempenho de Karnofsky. Quanto à formação dos autores, sete ${ }^{16-20,22-23}$ estudos foram publicados por profissionais da medicina; dois ${ }^{(13-14)}$ da psicologia e medicina, um da psicologia ${ }^{(15)} \mathrm{e} \mathrm{um}^{(21)} \mathrm{da}$ enfermagem. Devido à natureza do tema apresentado, bem como ao delineamento metodológico dos estudos incluídos, observou-se que todos os artigos analisados se enquadravam ao NE VI (Quadro 2.).
Quanto aos participantes dos estudos, identificaram-se as seguintes características: análise comparativa da QV após o Tx renal com grupos em HD e/ou sadios $(16,18-19,21,23)$ análise comparativa da $Q V$ após o Tx renal de um único grupo no pré e pós procedimento ${ }^{(17,20)}$; análise comparativa da QV após o Tx renal tardio entre dois grupos ${ }^{(13)}$; análise comparativa da QV após o Tx renal entre dois grupos, relacionada ao tipo de doação ${ }^{(14)}$; análise da QV de um único grupo apenas após o Tx renal ${ }^{(15)}$; análise comparativa da QV após o Tx renal entre dois grupos, relacionada com a idade dos participantes ${ }^{(22)}$.

Quadro 2. Distribuição dos artigos segundo título, questionário, autores, formação, periódico, ano e nível de evidência.

\begin{tabular}{|c|c|c|c|c|c|}
\hline Título do artigo & Questionário & Autores & Formação & Periódico / ano & $\begin{array}{l}\text { Nível de } \\
\text { evidência }\end{array}$ \\
\hline $\begin{array}{l}\text { Prospective Changes in Health-Related Quality } \\
\text { of Life and Emotional Outcomes in Kidney Trans- } \\
\text { plantation over } 6 \text { Years }\end{array}$ & SF-36; *TxEQ & Griva et al(13) & $\begin{array}{l}\text { Psicololgia / } \\
\text { medicina }\end{array}$ & $\begin{array}{l}\text { Journal of Transplanta- } \\
\text { tion / } 2011\end{array}$ & VI \\
\hline $\begin{array}{l}\text { Quality of life and emotional responses in cada- } \\
\text { ver and living related renal transplant recipients }\end{array}$ & SF-36; *TXEQ & Griva et al(14) & $\begin{array}{l}\text { Psicololgia / } \\
\text { medicina }\end{array}$ & $\begin{array}{l}\text { Nephrol Dial Trans- } \\
\text { plant / } 2002\end{array}$ & VI \\
\hline $\begin{array}{l}\text { Evolución de la calidad de vida relacionada con } \\
\text { la salud en los trasplantados renales }\end{array}$ & $\begin{array}{l}S F-36 ;{ }^{* *} E Q- \\
5 D ; * * * E S R D- \\
S C L\end{array}$ & $\begin{array}{l}\text { San Gregorio et } \\
\text { al(15) }\end{array}$ & Psicologia & Nefrología / 2007 & VI \\
\hline $\begin{array}{l}\text { Life situation and quality of life in young adult } \\
\text { kidney transplantat recipients }\end{array}$ & SF-36 & Aesebo et al(16) & Medicina & $\begin{array}{l}\text { Nephrol Dial Trans- } \\
\text { plant / } 2009\end{array}$ & VI \\
\hline $\begin{array}{l}\text { Changes in Health-Related Quality of Life in } \\
\text { Greek Adult Patients } 1 \text { Year After Successful } \\
\text { Renal Transplantation }\end{array}$ & SF-36 & $\begin{array}{l}\text { Balaska et } \\
\text { al(17) }\end{array}$ & Medicina & $\begin{array}{l}\text { Experimental and Cli- } \\
\text { nical Transplantation } \\
\text { / } 2006\end{array}$ & VI \\
\hline $\begin{array}{l}\text { Quality of life in renal transplant patients: } \\
\text { impact of a functioning graft }\end{array}$ & WHOQOL & $\begin{array}{l}\text { Bittencout et } \\
\text { al(18) }\end{array}$ & Medicina & $\begin{array}{l}\text { Rev Saúde Pública / } \\
2004\end{array}$ & VI \\
\hline $\begin{array}{l}\text { Qualidade de vida relacionada à saúde em } \\
\text { paciente transplantado renal }\end{array}$ & SF-36. & $\begin{array}{l}\text { Pereira et } \\
\text { al(19) }\end{array}$ & Medicina & J Bras Nefrol / 2003 & VI \\
\hline $\begin{array}{l}\text { Qualidade de vida e estratégias de enfrentamen- } \\
\text { to em pacientes submetidos a transplante renal }\end{array}$ & SF-36 & $\begin{array}{l}\text { Ravagnani } \\
\text { LMB, Domingos } \\
\text { NAM, Miyazaki } \\
\text { MCOS(20) }\end{array}$ & Medicina & $\begin{array}{l}\text { Estudos de Psicologia } \\
\text { / } 2007\end{array}$ & VI \\
\hline $\begin{array}{l}\text { Comparando a qualidade de vida de pacientes } \\
\text { em hemodiálise e pós-transplante renal pelo } \\
\text { "WHOQOL-BREF" }\end{array}$ & WHOQOL-Bref & $\begin{array}{l}\text { Farias GM, } \\
\text { Mendonça } \\
\text { AE0(21) }\end{array}$ & Enfermagem & $\begin{array}{l}\text { REME - Rev. Min. } \\
\text { Enferm. / } 2009\end{array}$ & VI \\
\hline $\begin{array}{l}\text { Graft and Quality of Life Outcomes in Older } \\
\text { Recipients a Kidney Transplant }\end{array}$ & SF-36 & Humar et al(22) & Medicina & $\begin{array}{l}\text { Experimental and Cli- } \\
\text { nical Transplantation } \\
\text { / } 2003\end{array}$ & VI \\
\hline $\begin{array}{l}\text { Health-Related Quality of Life in Emotionally } \\
\text { Related Kidney Transplantation: Deductions } \\
\text { from a Comparative Study }\end{array}$ & $\begin{array}{l}\text { Escala de Status } \\
\text { de Desempenho } \\
\text { de Karkofski }\end{array}$ & $\begin{array}{l}\text { Arogundade FA, } \\
\text { Abd-Essamie } \\
\text { MA, Barsoum } \\
\text { RS(23) }\end{array}$ & Medicina & $\begin{array}{l}\text { Saudi Journal of } \\
\text { Kidney Diseases and } \\
\text { Transplantation / } 2005\end{array}$ & VI \\
\hline
\end{tabular}

*Transplant Effects Questionnaire; **Euroqol-5D; ***End-Stage Renal Diesease Symptom Cheklist-Transplantation Module. Fonte: os autores, 2013.

Relativamente às evidências na melhoria da QV dos pacientes após o Tx renal, constatou-se na maioria dos estudos que, de modo geral, o Tx melhora expressivamente a $Q V$ do paciente com DRC, sendo prevalente a melhoria relacionada com o domínio físico(14-15,17-19). Observou-se também que o Tx melhora de modo significativo a QV emocional do paciente ${ }^{(13-14,17)}$. A análise dos estudos possibilitou ainda, trazer à luz do conhecimento 
que a QV do paciente após o Tx renal evolui com melhora importante, sendo semelhante à $\mathrm{QV}$ de voluntários sadios $^{(19)}$, porém, ainda com escores inferiores quando comparada com este grupo ${ }^{(16)}$. Alguns autores relatam que a $Q V$ do paciente com DRC após o Tx melhora consideravelmente em todos os aspectos/domínios ${ }^{(21,23)}$, sendo a idade do receptor e tipo de doação citados como fatores, praticamente, condicionantes ${ }^{(17,22)}$.

Em contrapartida, fatores relacionados ao meio ambiente, aparência e imagem corporal, medicação contínua, internações hospitalares e consultas rotineiras são elencados como os principais determinantes para diminuição da QV destes pacientes, mesmo com a realização do $\operatorname{Tx}^{(18,20)}$. Além disso, identificou-se que sentimentos de culpa em relação ao doador e preocupação com o enxerto podem influenciar negativamente a QV do paciente renal crônico transplantado ${ }^{(13-14)}$. Todas essas questões são citadas como as principais causas de baixos escores de dimensões/domínios referentes à relação social| ${ }^{16,18)}$, meio ambiente ${ }^{18}$, nível de dependência ${ }^{(13-14,20)}$ e aspectos psicológicos ${ }^{(13-14)}$.

\section{Discussão}

Estudos que buscam realizar a mensuração da QV da pessoa enferma apresentam-se em números elevados na literatura. Quando os sujeitos são doentes crônicos, tais estudos tornam-se ainda mais notáveis ${ }^{(24)}$. Isso, porque 0 referido público apresenta condições clínicas que perduram por longa data, comprometendo significativamente a promoção e manutenção de sua $\mathrm{QV}{ }^{(25)}$. Dessa maneira, muitos métodos analíticos foram desenvolvidos por diversos grupos de pesquisadores. Nesta revisão, o SF-36 foi identificado como o questionário com maior frequência de utilização nos estudos analisados. Acredita-se, que tal predomínio se deve ao fato de que este questionário seja de fácil compreensão para os pesquisadores e, principalmente, para os sujeitos - muitas vezes leigos - de diversos estudos, o que amplia sua aplicabilidade ${ }^{(24,26)}$. Vale lembrar ainda, que o referido instrumento é constituído por dimensões que avaliam a qualidade de vida relacionada à saúde (QVRS) de modo bastante abrangente, abarcando dimensões relacionadas à capacidade funcional, dor, estado geral de saúde, vitalidade, aspectos físicos, sociais, emocionais e relacionados à saúde mental do paciente ${ }^{(26)}$. Cabe ressaltar que outros instrumentos, tais como os específicos para avaliação da QV do paciente com DRC, bem como o instrumento proposto pela Organização Mundial de Saúde (OMS) e a Escala de Karnofski, identificados nesta revisão, apresentam-se igualmente válidos pela literatura internacional ${ }^{(18,21,23)}$.
Apesar da existência e validação científica de diversos instrumentos que permitem avaliar a QV do paciente com DRC, evidenciou-se na literatura que, em nefrologia, o grupo foco para a utilização de tais instrumentos mostra-se, predominantemente, composto por doentes renais crônicos em tratamento hemodialítico, cujos possíveis motivos para tal evidência já foram citados nesta revisão. Todavia, pesquisadores destacam ser de extrema importância avaliar a QVRS de portadores de DRC em diferentes estágios da doença, pois, contribui para um melhor acompanhamento clínico e possibilita que a assistência seja efetivada de maneira mais direciona$\mathrm{da}$, favorecendo a adoção de condutas terapêuticas com interferência mínima sobre a QV destes pacientes ${ }^{(27)}$. Assim, o desenvolvimento desta revisão possibilitou evidenciar a existência de lacunas na literatura no que diz respeito à difusão do conhecimento sobre estudos que objetivam avaliar a QV do paciente com DRC após o Tx renal, sendo as evidências confirmadas até mesmo por autores que realizam esses estudos ${ }^{(20)}$. Tais lacunas são ainda maiores, quando se avalia a produção científica da enfermagem sobre a temática em questão (quadro 2). Diante dessas evidências, entende-se haver necessidade de estímulo a grupos de pesquisas, em especial na enfermagem, para que haja maior produção de trabalhos sobre a QV do paciente com DRC no pós Tx.

Entretanto, é igualmente necessário o incentivo a estudos que destacam informações acerca dos cuidados aos pacientes transplantados, enfatizando os principais aspectos e resultados positivos e negativos de intervenções, assim como as dificuldades encontradas na prática para elaboração de estratégias de promoção e manutenção da QV desses pacientes. Para tanto, é imprescindível que seja minimizada a linha de distanciamento entre teoria e prática, pesquisa e cuidado(10). Tais estudos são essenciais para o fortalecimento da PBE, uma vez que podem constituir-se como uma bússola aos profissionais, fundamentando condutas e tomada de decisão sobre 0 planejamento assistencial para acrescentar qualidade à vida do paciente assistido. Com isto, sugere-se que mais estudos com NE V, VI e VII sejam produzidos, pois, somente a partir disso, será possível realizar recomendações sólidas sobre condutas e intervenções assistenciais válidas para a PBE que caracterizem em melhoria efetiva na QV do paciente com DRC pós Tx.

As evidências disponíveis na literatura apontam para uma melhora significativa na QV do paciente com DRC pós Tx renal, principalmente no domínio "físico". Infere-se que isso se deve ao fato deste domínio estar intimamente relacionado com a melhora das funções orgânicas que ocorre 
no paciente após o $\mathrm{Tx}^{(18-19)}$. Muitos estudiosos relatam que ter um rim transplantado funcionante faz com que o paciente vivencie fortes sentimentos emocionais e de bem estar geral relacionado à saúde ${ }^{(13-15,18-19)}$. Nesse mesmo contexto, a evidência de boa funcionalidade do rim torna-se ainda mais forte quando há doação de órgão por parte de doadores relacionados, ou seja, que possuem algum grau de parentesco com o receptor ${ }^{(14,17)}$. Nessa direção, estudos ainda apontam evidências de QV estatisticamente melhor quando o Tx renal ocorre em pacientes com idade máxima de 30 anos $^{(17)}$. Porém, alguns autores ressaltam que idade avançada, unicamente, deve ser desconsiderada como um fator condicionante para a não realização do Tx renal, pois, pacientes mais velhos também são capazes de alcançar níveis de $Q V$ após o Tx com escores de avaliação elevados ${ }^{(22)}$.

A comprovação científica de que o Tx renal apresenta muito mais benefícios para a QV do paciente com DRC em relação aos aspectos negativos, não é sinônimo da inexistência desses aspectos. Destarte, as condutas da equipe com o objetivo de minimizar os aspectos que influenciam negativamente na $\mathrm{QV}$ do paciente pós após o Tx renal deve ser implementada antes mesmo do procedimento. À enfermagem, cabe realizar educação em saúde ao paciente, cuidador e familiar ainda no pré-operatório, a fim de expor todas as variáveis relevantes do trans e pós-operatório e, principalmente, colocar quais as responsabilidades a serem assumidas no pós-operatório, enfatizando as questões relativas aos cuidados com a medicação, riscos de infecções oportunistas devido à imunossupressão, necessidade de acompanhamento ambulatorial contínuo, sinais e sintomas de rejeição, entre outras.

Além disso, é preciso realizar o levantamento dos diagnósticos de enfermagem inerentes a cada paciente, bem como estabelecer um plano de cuidados individual, que buscará atender a todas as necessidades do paciente, cuidadores e familiares. A enfermagem tem, ainda, a função de articular a interação entre paciente, cuidador e familiar com a equipe multiprofissional ${ }^{(28)}$. A esta, cabe oferecer assistência especializada nos diversos contextos do cuidado e buscar, através do trabalho interdisciplinar, (re) conhecer os fatores de risco à QV do paciente com DRC após o Tx renal para, então, implementar ações multiassistenciais que viabilizem a promoção e manutenção da QV desses pacientes.

\section{Conclusão}

As evidências apontam que os anseios do paciente portador de DRC em HD pela realização de um Tx renal justificam-se no que diz respeito à busca por melhoria na $Q V$, pois, confirmou-se que o Tx é responsável pelo adicionamento de qualidade à vida do paciente pós procedimento. Foi possível, ainda, identificar as facetas relacionadas à melhor QV pós Tx renal, sendo que estas dizem respeito, predominantemente, à dimensões/ domínios "físico" e "emocional". Contudo, verificou-se que os valores dos escores de avaliação da QV no pós Tx renal podem depender de alguns fatores, tais como idade do receptor e tipo de doação. Já os fatores relacionados com as relações sociais, meio ambiente, nível de dependência e aspectos psicológicos emergiram como as principais causas de baixos escores de avaliação da QV no pós Tx.

A realização deste estudo, tornou possível evidenciar a existência de lacunas na literatura no que diz respeito à produção científica sobre a temática abordada, principalmente, estudos provenientes de profissionais da enfermagem. Diante disso, sugere-se que futuras pesquisas sejam realizadas a fim de estabelecer maiores evidências sobre os benefícios do Tx à $Q V$ do paciente com DRC, em especial aqueles que apresentem a interação multiprofissional nas busca pela implementação de intervenções com interferência mínima na QV do paciente no pós Tx renal. Com isso, certamente será possível corroborar a PBE, uma vez que tais estudos servirão como norteadores para tomadas de decisões profissionais e elaboração de estratégias de promoção e manutenção da QV do paciente no pós Tx renal.

Finalmente, considera-se necessário que a equipe de enfermagem desempenhe o papel de educação em saúde junto dos pacientes, cuidadores e familiares ainda no pré-operatório, preparando-os e informando-os sobre suas responsabilidades, bem como esclarecendo quaisquer dúvidas. Além disso, a enfermagem deve estabelecer a linha de comunicação entre paciente, cuidador e familiar com a equipe multiprofissional. Já esta, deve estar em contínuo estudo interdisciplinar na busca pelo atendimento a todas as necessidades dos pacientes, cuidadores e familiares, a fim de estabelecer um planejamento assistencial que objetive erradicar ou minimizar os pontos que influenciam negativamente na QV do paciente transplantado renal. 


\section{Referências}

1. Nobre MRC, Bernardo WM, Jatene FB. A prática clínica baseada em evidências: Parte III Avaliação crítica das informações de pesquisas clínicas. Rev. Assoc. Med. Bras.[Internet] 2004 [citado 2013 Jan. 20]; 50 (2): 221-8. Disponível em: http:// www.scielo.br/pdf/ramb/v50n2/20787.pdf.D0I. org/10.1590/S0104-42302004000200042.

2. Domenico EBLD, Ide CAC. Enfermagem baseada em evidências: princípios e aplicabilidades. Rev. Latino-am. Enfermagem. [Internet] 2003 [citado 2013 Jan. 20]; 11(1): 115-8. Disponível em: http://www.scielo.br/pdf/rlae/v1ln1/16568.pdf. D0I.org/10.1590/S0104-11692003000100017.

3. International Council of Nurses (ICN). The ICN code of ethics for nurses. [Internet] Geneva, Switzerland; 2012 [cited 2013 Jan. 20]. Available from: http:// www.abennacional.org.br/images/conteudo/file/ ICN\%20Code\%20of\%20Ethics\%202012.pdf.

4. Campos Mo, Neto JFR. Doenças crônicas não transmissíveis: fatores de risco e repercussão na qualidade de vida. Rev. Baiana Saúde Pública. [Internet] 2009 [citado 2013 Jan. 20]; 33 (4): 561-581. Disponível em: http://inseer.ibict.br/rbsp/ index.php/rbsp/article/viewFile/289/pdf_102.

5. Carvalho GMC, Lima FET, Barbosa IV, et al. Estudos brasileiros sobre nefrologia nas teses e dissertações de enfermagem. Rev. bras. enferm. [Internet] 2010 [citado 2013 Jan. 20]; 63(6): 1052-1055. Disponível em: http://www.scielo.br/ pdf/reben/v63n6/28.pdf.D0I.org/10.1590/S003471672010000600028.

6. Pennafort VPS, Furtado AM, Fialho AVM, et al. Produção do conhecimento científico de Enfermagem em Nefrologia. Rev. bras. enferm. [Internet] 2010 [citado 2013 Jan. 20]; 63 (5): 830-6. Disponível em: http://www.scielo.br/pdf/reben/v63n5/22.pdf.D0I. org/10.1590/S0034-71672010000500022.

7. Bertolin DC, Pace $A E$, Kusumota $L$, et al. . Ways for people on hemodialysis to deal with stressors: a literature review. Acta paul. enferm. [Internet] 2008 [cited 2013 Jan. 20]; 21 (n. spe): 179-86. Available from: http://www.scielo.br/pdf/ ape/v2lnspe/en_a08v2lns.pdf.D0I.org/10.1590/ S0103-21002008000500008.
8. Meireles VC, Goes HLF, Dias TA. Vivência do paciente renal crônico em tratamento hemodialítico: subsídios para o profissional enfermeiro. Ciência, cuidado e saúde. [Internet] 2004 [citado 2013 Jan. 20]; 3 (2): 169-178. Disponível em: http:// eduem.uem.br/ojs/index.php/CiencCuidSaude/article/viewFile/5423/3475.

9. Galvão CM, Sawada N0, Trevizan MA. Revisão sistemática: recurso que propicia a incorporação das evidências na prática da enfermagem. Rev. latinoam Enfermagem. [Internet] 2004 [citado 2013 Jan. 21]; 12 (3): 549-56. Disponível em: http:// www.scielo.br/pdf/rlae/v12n3/v12n3al4.pdf.D0I. org/10.1590/S0104-11692004000300014.

10. Mendes KDS, Silveira RCCP, Galvão CM. Revisão integrativa: método de pesquisa para a incorporação de evidências da saúde e na enfermagem. Texto contexto-enferm. [Internet] 2008 [citado 2013 Jan. 21]; 17 (4): 758-64. Disponível em: http://www.scielo.br/pdf/tce/v17n4/18.pdf.D0I. org/10.1590/S0104-07072008000400018.

11. Souza MT, Silva MD, Carvalho R. Integrative review: what is it? How to do it?. Einstein (São Paulo). [Internet] 2010 [cited 2013 Apr. 10]; 8(1 Pt 1):102-6. Available from: http://apps. einstein.br/revista/arquivos/PDF/1134-Einsteinv8n1p102-106.pdf.

12. Galvão CM. Evidences hierarchies. Acta Paul Enferm. [Internet] 2006 [cited 2013 Apr. 10]; 19 (2): VI. Available from: http://www.scielo.br/pdf/ ape/v19n2/en_a01v19n2.pdf.

13. Griva K, Stygall J, Hui Ng J, et al. Prospective Changes in Health-Related Quality of Life and Emotional Outcomes in Kidney Transplantation over 6 Years. Journal of Transplantation. [Internet] 2011 [cited 2013 Feb. 03]: 1-12. Available from: http:// www.hindawi.com/journals/jtran/2011/671571/. D0I:10.1155/2011/671571.

14. Griva K, Ziegelmann JP, Thompson D, et al. Quality of life and emotional responses in cadaver and living related renal transplant recipients. Nephrol Dial Transplant. [Internet] 2002 [cited 2013 Feb 03]; 17 (2): 2204-2211. Available from: http://ndt. oxfordjournals.org/content/17/12/2204.full.pdf. D0I: 10.1093/ndt/17.12.2204. 
15. San Gregorio MAP, Rodríguez AM, Domínguez RD, et al. Evolución de la calidad de vida relacionada con la salud en los trasplantados renales. Nefrología. [Internet] 2007 [citado 2013 Feb. 03]; 27 (5): 619-626. Disponible en: http://revistanefrologia.com/revistas/ P1-E260/P1-E260-S132-A4855.pdf.

16. Aesebo W, Homb-Vesteraas AN, Hartmann A, et al. Life situation and quality of life in young adult kidney transplant recipients. Nephrol Dial Transplant. [Internet] 2009 [cited 2013 Feb. 03] 24: 304308. Available from: http://ndt.oxfordjournals.org/ content/early/2008/10/07/ndt.gfn537.full.pdf.D0I: 10.1093/ndt/gfn537.[Epub ahead of print].

17. Balaska A, Moustafellos $P$, Gourgiotis $S$, et al. Changes in Health-Related Quality of Life in Greek Adult Patients 1 Year After Successful Renal Transplantation. Experimental and Clinical Transplantation. [Internet] 2006 [cited 2013 Feb. 03]; 4 (2): 521-524. Available from: http://www.ectrx.org/ forms/ectrxcontentshow.php?year $=2006 \&$ volume $=$ $4 \&$ issue $=2 \&$ supplement $=0 \&$ makale_no $=0 \&$ spage number $=521 \&$ content_type $=$ FULL\%20TEXT.

18. Bittencout ZZLC, Alves Filho G, Mazzalli M, et al. Quality of life in renal transplant patients: impact of a functioning graft. Rev Saúde Pública. [Internet] 2004 [cited 2013 Feb. 04]; 38 (5):732-734. Available from: http://www.scielo.br/pdf/rsp/v38n5/en_21764. pdf.DOI.org/10.1590/S0034-89102004000500018.

19. Pereira LC, Chang J, Fadil-Romão MA, et al. Qualidade de vida relacionada a saúde em paciente transplantado renal. J Bras Nefrol. [Internet] 2003 [citado 2013 Fev. 05]; 25 (1): 10-16. Disponível em: http://www.jbn.org.br/detalhe_artigo.asp?id=418.

20. Ravagnani LMB, Domingos NAM, Miyazaki MCOS. Qualidade de vida e estratégias de enfrentamento em pacientes submetidos a transplante renal. Estudos de Psicologia. [Internet] 2007 [citado Fev. 15]; 12 (2): 177-184. Disponível em: http://www. scielo.br/pdf/epsic/v12n2/a10v12n2.pdf.

21. Farias GM, Mendonça AEO. Comparando a qualidade de vida de pacientes em hemodiálise e póstransplante pelo "WHOQOL_BREF". Reme - Rev. Min. Enferm. [Internet] 2009 [citado 2013 Fev. 15]; 13 (4): 574-583. Disponível em: http://www. enf.ufmg.br/site_novo/modules/mastop_publish/ files/files_4c1220c4cae6d.pdf.
22. Humar A, Denny R, Matas AJ, et al. Graft and Quality of Life Outcomes in Older Recipients of a Kidney Transplant. Experimental and Clinical Transplantation. [Internet] 2003 [cited 2013 Feb. 15]; 1 (2): 69-72. Available from: http://www.ectrx.org/ forms/ectrxcontentshow.php?year $=2003 \&$ volume $=$ $1 \&$ issue $=2 \&$ supplement $=0 \&$ makale_no $=0 \&$ spage number $=69 \&$ content_type=FULL\%20TEXT.

23. Aroundade FA, Abd-Essamie MA, Barsoum RS. Health-Related Quality of Life in Emotionally Related Kidney Transplantation: Deductions from a Comparative Study. Saudi Journal of Kidney Diseases and Transplantation. [Internet] 2005 [cited 2013 Feb. 15]; 16 (3): 311-320. Available from: http://www.sjkdt.org/temp/SaudiJKidneyDisTranspl163311-369308_101530.pdf.

24. Duarte PS, Miyazaki MCOS, Ciconelli RM, et al. Tradução e adaptação cultural do instrumento de avaliação de qualidade de vida para pacientes renais crônicos (KDQOL-SFTM). Rev. Assoc. Med. Bras. [Internet] 2003 Dez [citado 2013 Mar. 12]; 49(4): 375-81. Disponível em: http://www.scielo. br/pdf/ramb/v49n4/18335.pdf.D0I.org/10.1590/ S0104-42302003000400027.

25. Rudnick T. Preditores de qualidade de vida em pacientes renais crônicos. Estud. Psicol. [Internet] 2007 [citado 2013 Mar. 12]; 24(3):343-55. Disponível em: http://www.scielo.br/pdf/estpsi/v24n3/a06v24n3.pdf. D0I.org/10.1590/S0103-166X2007000300006.

26. Cruz MC, Andrade $C$, Urrutia $M$, Draibe $S$, Nogueira-Martins LA, Sesso RCC. Quality of life in patients with chronic kidney disease. Clinics. [Internet] 2011[cited Mar. 14]; 66(6):991-995. Available from: http://www.scielo.br/pdf/clin/v66n6/12.pdf. D0I.org/10.1590/S1807-59322011000600012.

27. Pagels AA, Soderkvist BK, Medin $C$, et al. Healthrelated quality of life in different stages of chronic kidney disease and at initiation of dialysis treatment. Health and quality of life outcomes. [Internet] 2012 [cited 2013 Mar. 14]; 10(71):1-11. Available from: http://www.ncbi.nlm.nih.gov/pmc/articles/ PMC3511211. D0I:10.1186/1477-7525-10-71.

28. Carpenito LJ. Planos de Cuidados de Enfermagem e Documentação: Diagnósticos de enfermagem e Problemas Colaborativos. $2^{\mathrm{a}}$ ed. Porto Alegre: Artmed; 1999. 